\title{
ÚNG DỤNG CÔNG NGHỆ THÔNG TIN TRONG CÔNG TÁC ĐÁNH GIÁ Độ CHÍNH XÁC SỐ LIỆU ĐO SÂU ĐỚN TIA PHỤC VỤ THÀNH LẬP BẢN ĐỒ ĐỊ̂A HÌNH ĐÁY BIỂN
}

\author{
NGUYẼ̃N VĂN CƯƠNG (1), NGUYẼ̃N GIA TRỌNG ${ }^{(2)}$, NGUYẼ̃N THẾ HIỆP(2), \\ ĐẶNG VIẾT HOAN ${ }^{(3)}$, PHẠM THỊ PHƯƠNG LIÊN ${ }^{(4)}$ \\ ${ }^{(1)}$ Cuc Quản lý khai thác biển và hải đảo, Tổng cuc Biển và Hải đảo Việt Nam \\ ${ }^{(2)}$ Khoa Trắc địa - Bản đồ và Quản lý đất đai, Trương Đại học Mỏ - Địa chất \\ ${ }^{(3)}$ Đoàn đo đạc, biên vẽ hải đồ và nghiên cứu biển \\ ${ }^{(4)}$ Bộ Tài nguyên và Môi trường
}

\section{Tóm tắt:}

Đặc thù khi đo sâu phục vụ thành lập bản đồ địa hình đáy biển đó là người đi đo không quan sát trực tiếp được địa hình khu đo. Để phản ánh đủng địa hình đáy biển đòi hỏi phải đo các điểm chi tiết với mật độ dày đặc sư dụng công nghệ đo sâu hồi âm hoặc 1 số công nghệ đo duới nước khác. Tuy nhiên, dù đã đo dày đặc thì bên cạnh các đuờng đo sâu cần phải đo thêm các đường kiểm tra. Sau khi đã có số liệu đo sâu và số liệu kiểm tra cần phải kiểm tra giá trị độ lệch giữa độ sâu xác định theo đương kiểm tra với độ sâu xác định theo đường đo sâu. Các tác giả đã Trên co sở tìm hiểu các quy định kỹ thuật về đánh giá kết quả đo sâu bằng máy đo sâu hồi âm đơn tia, chúng tôi đã để tù đó lựa chọn thuật toán cũng nhu lự chọn ngôn ngũ lập trình để xây dựng chuoong trình kiểm tra số liệu đo sâu. Bài báo giới thiệu kết quả lựa chọn thuật toán và chuong trình kiểm tra dũ liệu đo sâu đã được xây dụng. Với kết quả kiểm tra số liệu đo sâu hồi âm đơn tia có so sánh, đối chưng với kết quả của các công trình đã thi công cho thấy chuơng trình được xây dựng hoàn toàn đáp úng yêu cầu kiểm tra số liệu đo sâu bằng máy đo sâu hồi âm đơn tia.

\section{Mở đầu}

Việt Nam là một quốc gia có hơn $3000 \mathrm{~km}$ đường bờ biển, diện tích biển lên tới 1 triệu km² và mạng lưới sông ngòi dày đặc nên công tác đo sâu phục vụ đo vẽ bản đồ địa hình đáy biển nói riêng và các công tác trắc địa dưới nước, thành lập các bản đồ hàng hải, phục vụ giao thông đường thủy nội địa... nói chung là công việc hết sức cần thiết. Công tác đo sâu trong đo vẽ địa hình đáy biển và đo vẽ bản đồ địa hình đáy biển phục vụ khảo sát thiết kế và thi công xây dựng các công trình biển là công việc phức tạp do phải làm việc trong môi trường luôn biến động, chúng ta không nhận thức được mức độ phức tạp của địa hình bằng các giác quan mà phải nhận biết bằng máy móc thiết bị. Khi thành lập bản đồ địa hình trên đất liền, người đo có thể đo chi tiết đúng vào các điểm đặc trưng, song khi đo địa hình đáy biển do không thể quan sát được dáng địa hình đáy biển bằng mắt nên người ta không thể thực hiện được điều này. Để phản ánh được trung thực bề mặt địa hình dưới nước, đòi hỏi phải sử dụng các thiết bị đo đạc có khả năng đo với mật độ dày đặc các điểm. Công nghệ được lựa chọn để thực hiện được điều này là các máy đo sâu hồi âm (đơn tia, đa tia). Tuy nhiên, do không thể trực tiếp quan sát được bề mặt địa hình dưới mặt nước nên việc đo dày đặc các điểm là chưa đủ mà cần phải thiết kế các đường đo sâu kiểm tra, xác thực kết quả đo. Các quy định kỹ thuật về thiết kế đường đo sâu, đường đo kiểm tra được quy định rất cụ thể bởi các cơ quan có thẩm quyền Nhà nước [1], [2] và [3]. Sau khi đã có số liệu đo sâu, số liệu đo kiểm tra cần phải tính toán xác định độ lệch độ sâu giữa độ sâu xác định theo đường đo sâu với độ sâu xác định được theo đường kiểm tra

Ngày nhận bài: 05/6/2021, ngày chuyển phản biện: 09/6/2021, ngày chấp nhận phản biện: 15/6/2021, ngày chấp nhận đăng: 9/7/2021 
rồi so sánh với quy định về độ lệch độ sâu được quy định trong các văn bản pháp quy [1], [2] và [3].

Công tác nghiên cứu liên quan đến công nghệ và kỹ thuật đo sâu đã được tiến hành bài bản trên thế giới để sau đó được cụ thể hóa thành quy trình kỹ thuật cũng như công nghệ khép kín. Tại Việt Nam, cũng đã có một số nghiên cứu nhằm ứng dụng có hiệu quả công tác đo sâu hồi âm phục vụ các mục tiêu khác nhau.

Vũ Hồng Tập [6] đã nghiên cứu về ảnh hưởng của vận tốc âm đến kết quả đo sâu trong thành lập bản đồ địa hình đáy biển. Tác giả Nguyễn Văn Cương [5] đã nghiên cứu quy luật biến đổi vận tốc âm tại khu vực Vịnh Bắc Bộ để từ đó đề xuất hoàn thiện quy trình lấy mẫu vận tốc âm.

Các tác giả Dương Quốc Lương [7], Tăng Quốc Cương [9] và Phạm Vũ Vinh Quang [8] đã nghiên cứu các giải pháp ứng dụng máy đo hồi âm đơn tia, máy đo vận tốc âm, công nghệ dò thủy âm quét sườn trong thành lập bản đồ địa hình đáy biển.

Tính đến thời điểm hiện tại, các quy định kỹ thuật về kiểm tra số liệu đo sâu, đặc biệt là đo sâu hồi âm đơn tia đã tương đối hoàn chỉnh nhưng chưa có công bố nào về thuật toán cũng như xây dựng chương trình kiểm tra dữ liệu đo sâu hồi âm đơn tia phục vụ thành lập địa hình đáy biển. Xuất phát từ thực tế đó, nhóm tác giả đã tìm hiểu các quy định kỹ thuật, lựa chọn thuật toán và ứng dụng công nghệ thông tin để xây dựng chương trình kiểm tra dữ liệu đo sâu sử dụng máy đo sâu hồi âm đơn tia. Các kết quả về lựa chọn thuật toán và xây dựng chương trình sẽ được trình bày cụ thể trong các phần sau.

\section{Căn cứ pháp quy và thuật toán xây dụ̣ng chương trình kiểm tra dữ liệu đo sâu}

\subsection{Căn cút pháp quy}

Các tiêu chí đánh giá độ chính xác đo sâu đơn tia phục vụ đo vẽ bản đồ địa hình đáy biển được tuân thủ theo các văn bản quy phạm pháp luật như sau: Quyết định số 180/1998/QĐ-ĐC ngày 31/3/1998 của Tổng cục Địa chính và các thông tư của Bộ Tài nguyên và Môi trường: số 03/2007/QĐ-BTNMT ngày 12/02/2007, số 04/2011/TT-BTNMT ngày 01/08/2011, số 34/2011/TTBTNMT ngày 01/08/2011, và số 63/2017/TT-BTNMT ngày 22/12/2017;

Dựa theo các tiêu chí đánh giá độ chính xác theo các quy định kỹ thuật hiện hành [1], [2], [3], bài báo lựa chọn thuật toán và xây dựng chương trình kiểm tra, đánh giá độ chính xác số liệu đo sâu đơn tia phục vụ thành lập bản đồ địa hình đáy biển.

\subsection{Thuật toán kiểm tra dĩ liệu đo sâu}

Để kiểm tra độ chính xác của dữ liệu đo sâu, cần thực hiện các nội dung công việc như sau: (1) xác định tọa độ của điểm giao cắt giữa đường đo sâu và đường kiểm tra, (2) nội suy độ sâu của điểm giao cắt và (3) tính độ lệch độ sâu giữa độ sâu xác định theo số liệu đo sâu và độ sâu xác định theo số liệu kiểm tra.

Để xác định tọa độ của điểm giao cắt, có thể sử dụng phương pháp giải tích thông qua lấy giao cắt của hai đường thẳng.

Giả sử tọa độ của 2 điểm thuộc đường đo sâu là $\mathrm{DS}_{1}\left(\mathrm{x}_{1} ; \mathrm{y}_{1} ; \mathrm{h}_{1}\right)$ và $\mathrm{DS}_{2}\left(\mathrm{x}_{2} ; \mathrm{y}_{2} ; \mathrm{h}_{2}\right)$; tọa độ tương ứng của 2 điểm thuộc đường kiểm tra là $\mathrm{KT}_{1}\left(\mathrm{x}_{1}^{\prime} ; \mathrm{y}_{1}^{\prime} ; \mathrm{h}_{1}^{\prime}\right)$ và $\mathrm{KT}_{2}\left(\mathrm{x}_{2}^{\prime} ; \mathrm{y}_{2}^{\prime} ; \mathrm{h}_{2}^{\prime}\right)$ thì tọa độ mặt bằng của điểm giao cắt $\mathrm{GC}\left(\mathrm{x}_{\mathrm{gc}} ; \mathrm{y}_{\mathrm{gc}}\right)$ được xác định như sau:

$$
\mathrm{L}_{1}=\frac{\left(\mathrm{y}_{2}-\mathrm{y}_{1}\right)}{\left(\mathrm{x}_{2}-\mathrm{x}_{1}\right)} ; \quad \mathrm{L}_{2}=\frac{\left(\mathrm{y}_{2}^{\prime}-\mathrm{y}_{1}^{\prime}\right)}{\left(\mathrm{x}_{2}^{\prime}-\mathrm{x}_{1}^{\prime}\right)}
$$




$$
\begin{aligned}
& \mathrm{C}_{1}=\mathrm{y}_{2}-\mathrm{L}_{1} \cdot \mathrm{x}_{1} ; \quad \mathrm{C}_{2}=\mathrm{y}_{2}^{\prime}-\mathrm{L}_{2} \cdot \mathrm{x}_{1}^{\prime} \\
& \mathrm{x}_{\mathrm{gc}}=\frac{\mathrm{C}_{2}-\mathrm{C}_{1}}{\mathrm{~L}_{2}-\mathrm{L}_{1}} ; \quad \mathrm{y}_{g c}=\mathrm{L}_{1} \cdot \mathrm{x}_{\mathrm{gc}}+\mathrm{C}_{1}
\end{aligned}
$$

Sau khi xác định được tọa độ của điểm giao cắt, độ sâu của điểm giao cắt theo số liệu đo sâu $\mathrm{h}_{\mathrm{ds}-\mathrm{gc}}$ và theo số liệu đo kiểm tra $\mathrm{h}_{\mathrm{ds}-\mathrm{kt}}$ được xác định theo phương pháp nội suy theo khoảng cách bởi công thức:

$$
\begin{aligned}
& \mathrm{h}_{\mathrm{gc}-\mathrm{ds}}=\mathrm{h}_{2} \frac{\mathrm{x}_{2}-\mathrm{x}_{\mathrm{gc}}}{\mathrm{x}_{2}-\mathrm{x}_{1}}+\mathrm{h}_{1} \frac{\mathrm{x}_{\mathrm{gc}}-\mathrm{x}_{1}}{\mathrm{x}_{2}-\mathrm{x}_{1}} \\
& \mathrm{~h}_{\mathrm{gc}-\mathrm{kt}}=\mathrm{h} \oint \frac{\mathrm{y} \frac{\phi}{2}-\mathrm{y}_{\mathrm{gc}}}{\mathrm{y} \phi-\mathrm{y} \phi}+\mathrm{h} \oint \frac{\mathrm{y}_{\mathrm{gc}}-\mathrm{y} \phi}{\mathrm{y} \ell-\mathrm{y} \phi}
\end{aligned}
$$

Độ lệch độ sâu theo đường đo sâu và đường kiểm tra được xác định theo công thức:

$$
\mathrm{dh}=\mathrm{h}_{\mathrm{gc}-\mathrm{ds}}-\mathrm{h}_{\mathrm{gc}-\mathrm{kt}}
$$

Sau khi xác định độ lệch độ sâu giữa giá trị nội suy theo đường đo sâu và giá trị tương ứng nội suy theo đường kiểm tra tại các điểm giao cắt, tiến hành thống kê phần trăm số lượng các điểm giao cắt có độ lệch theo như quy định và đánh giá độ chính xác xác định độ sâu của khu đo theo phương pháp hiệu trị đo kép

\section{Xây dựng chương trình kiểm tra dữ liệu đo sâu đơn tia}

Dựa trên các quy định hiện hành, thuật toán được lựa chọn như đã trình bày trong mục 2 , các tác giả lựa chọn ngôn ngữ lập trình Visual Studio.NET để xây dựng chương trình kiểm tra số liệu đo sâu được đo bằng máy đo sâu đơn tia. Giao diện chính của chương trình được cho như trong hình 1.

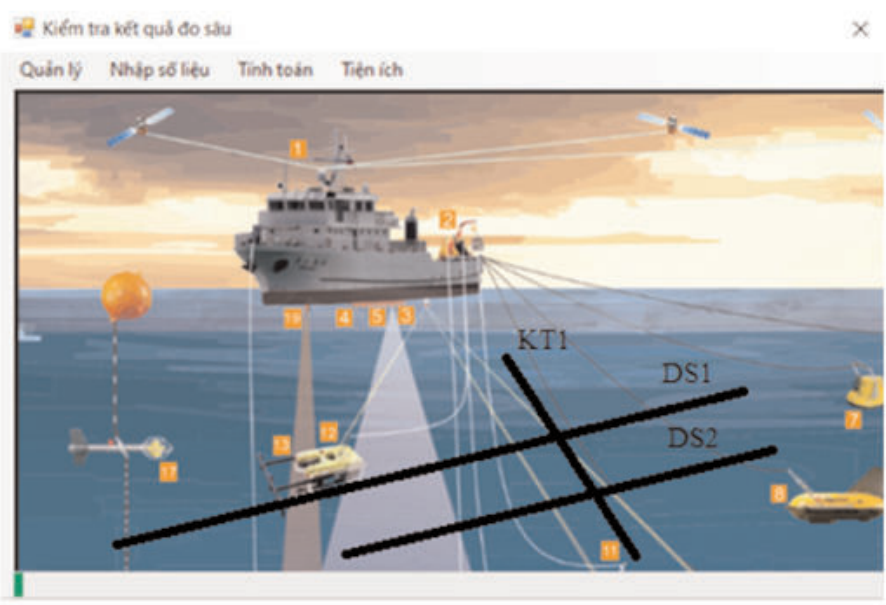

Hình 1: Giao diện chính của chuoong trình kiểm tra số liệu đo sâu

Từ hình 1 có thể thấy, chương trình có giao diện bằng tiếng Việt nên thân thiện với người sử dụng. Chương trình đã lập có các tính năng như sau:

- Quản lý: cho phép người sử dụng tạo dự án mới, sau khi dự án được tạo sẽ bao gồm các thư 
mục con chứa số liệu đầu vào và kết quả tính toán.

- Nhập số liệu: Nhập tệp số liệu đo sâu và tệp số liệu kiểm tra theo định dạng như trong hình 2.

\begin{tabular}{|c|c|c|c|c|c|c|c|}
\hline 1 & 2326343.65 & 452493.31 & -2.56 & 1 & 2326356.30 & 454928.28 & -8.69 \\
\hline 2 & 2325331.59 & 439672.36 & -6.01 & 2 & 2326347.25 & 454928.34 & -8.70 \\
\hline 3 & 2325322.45 & 439668.41 & -5.93 & 3 & 2326338.11 & 454928.22 & -8.70 \\
\hline 4 & 2325312.98 & 439665.11 & -5.77 & 4 & 2326329.08 & 454927.94 & -8.70 \\
\hline 5 & 2325303.58 & 439661.52 & -5.61 & 5 & 2326319.95 & 454927.53 & -8.70 \\
\hline 6 & 2325294.30 & 439657.75 & -5.43 & 6 & 2326310.82 & 454927.12 & -8.70 \\
\hline 7 & 2325284.81 & 439654.54 & -5.28 & 7 & 2326301.80 & 454926.74 & -8.71 \\
\hline $\begin{array}{l}8 \\
9\end{array}$ & $\begin{array}{r}2325275.33 \\
2325265.55\end{array}$ & $\begin{array}{l}439651.36 \\
439649.18\end{array}$ & $\begin{array}{l}-5.10 \\
-4.93\end{array}$ & 8 & 2326292.77 & 454926.60 & -8.71 \\
\hline 10 & 2325255.54 & 439648.85 & -4.71 & 9 & 2326283.62 & 454926.67 & -8.71 \\
\hline 11 & 2325245.74 & 439650.67 & -4.52 & 10 & 2326274.47 & 454926.86 & -8.72 \\
\hline 12 & 2325236.02 & 439653.26 & -4.32 & 11 & 2326265.42 & 454927.13 & -8.73 \\
\hline 13 & 2325226.68 & 439656.80 & -4.23 & 12 & 2326256.36 & 454927.41 & -8.74 \\
\hline 14 & 2325217.74 & 439661.37 & -4.16 & 13 & 2326247.25 & 454927.69 & -8.75 \\
\hline 15 & 2325209.12 & 439666.42 & -4.15 & 14 & 2326238.11 & 454927.84 & -8.76 \\
\hline 16 & 2325192.20 & 439677.13 & -4.53 & 15 & 2326229.01 & 454927.90 & -8.76 \\
\hline 17 & 2325184.08 & 439682.98 & -5.35 & 16 & 2326219.95 & 454927.97 & -8.77 \\
\hline 18 & 2325176.18 & $\begin{array}{l}439689.10 \\
439695.34\end{array}$ & -6.31 & 17 & 2326210.88 & 454928.00 & -8.77 \\
\hline $\begin{array}{l}19 \\
20\end{array}$ & $\begin{array}{l}2325168.34 \\
2325160.52\end{array}$ & $\begin{array}{l}439695.34 \\
439701.64\end{array}$ & $\begin{array}{l}-7.21 \\
-7.96\end{array}$ & 18 & 2326201.85 & 454927.97 & -8.76 \\
\hline \multicolumn{4}{|c|}{ Hình 2a. Số liệu đo sâu } & \multicolumn{4}{|c|}{ Hình 2b. Số liệu kiểm tra } \\
\hline
\end{tabular}

Sau khi số liệu được nhập, chương trình tự động phân tách dữ liệu của từng đường đo sâu và đường kiểm tra riêng rẽ nhau.

- Tính toán: Cho phép kiểm tra kết quả đo sâu với các tính năng cài đặt cho như trong hình 3.

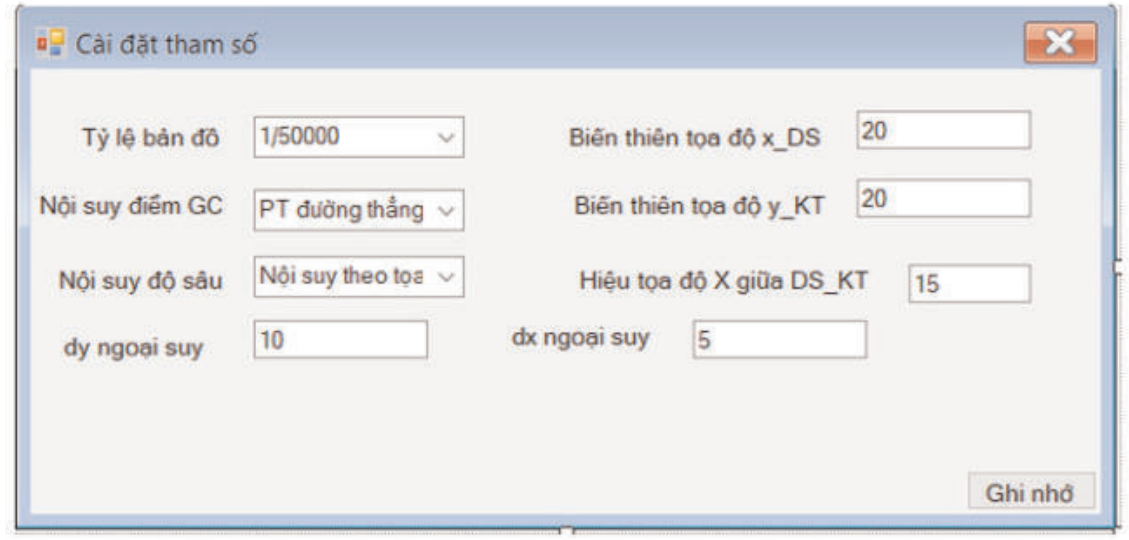

Hình 3: Cài đặt phương pháp tính toán khi kiểm tra kết quả đo sâu

Trong phần cài đặt cho phép lựa chọn tỷ lệ bản đồ cần kiểm tra, phương pháp nội suy điểm giao cắt cũng như phương pháp nội suy độ sâu của điểm giao cắt.

Sau khi chạy chương trình dạng số liệu đầu ra được thể hiện như bảng dưới:

Bảng thống kê các điểm được đánh giá theo dạng như sau:

\begin{tabular}{|c|c|c|c|c|c|c|c|c|c|c|c|}
\hline \multirow{2}{*}{ STT } & \multicolumn{3}{|c|}{ Điểm đo sâu } & \multirow{2}{*}{ STT } & \multicolumn{3}{|c|}{ Điểm đo kiểm tra } & \multicolumn{3}{|c|}{ Điểm nôi suy } & \multirow[b]{2}{*}{$\mathrm{DH}$} \\
\hline & $\mathrm{x}$ & $\mathrm{Y}$ & $\mathrm{H}$ & & $\mathrm{x}$ & $\mathrm{Y}$ & $\mathrm{H}$ & $\mathrm{x}$ & $\mathrm{Y}$ & $\mathrm{Hds} / \mathrm{Hkt}$ & \\
\hline 243 & 2325659 & 440133.1 & -9.17 & 17427 & 2325673 & 440124.8 & -8.97 & \multirow{2}{*}{2325666} & \multirow{2}{*}{440124.3} & -9.193 & \multirow{2}{*}{-0.207} \\
\hline 244 & 2325666 & 440125.3 & -8.99 & 17428 & 2325664 & 440124 & -9.02 & & & -8.986 & \\
\hline
\end{tabular}




\section{Tính toán thực nghiệm}

Với chương trình tính toán đã được xây dựng như giới thiệu trong mục 3 , tiến hành kiểm tra số liệu đo sâu với 03 khu vực với các tỷ lệ như sau:

+ Bản đồ tỷ lệ 1:50.000 khu vực thực nghiệm Ninh Thuận - Kiên Giang.

+ Bản đồ tỷ lệ 1:10.000 khu vực thực nghiệm Nha Trang - Khánh Hòa.

+ Bản đồ tỷ lệ 1:5.000 khu vực thực nghiệm Vân Đồn - Quảng Ninh.

Kết quả tính kiểm tra như sau:

\section{a. Với mảnh bản đồ tỷ lệ 1:5.000}

Tổng hợp kết quả độ lệch độ sâu giữa các tuyến đo sâu và đo kiểm tra như sau:

[1] Số điểm có độ sâu nội suy từ 0 đến $-30 \mathrm{~m}$ : 10198 điểm

1 Số điểm có $\mathrm{dh}$ từ $0 \mathrm{~m}$ đến $0.51 \mathrm{~m}$ : $\quad 9294$ điểm $\quad 91.14 \%$

2 Số điểm có dh từ $0.51 \mathrm{~m}$ đến $0.60 \mathrm{~m}$ : 145 điểm $\quad 1.42 \%$

3 Số điểm có dh lớn hơn $0.60 \mathrm{~m}: \quad 682$ điểm $\quad 6.69 \%$

[2] Số điểm có độ sâu nội suy nhỏ hơn -30m: 0 điểm

1 Số điểm có dh từ $0 \mathrm{~m}$ đến $0.51 \mathrm{~m}$ : $\quad 0$ điểm $\quad 0 \%$

2 Số điểm có dh từ $0.51 \mathrm{~m}$ đến $0.60 \mathrm{~m}$ : $\quad 0$ điểm $\quad 0 \%$

3 Số điểm có dh lớn hơn $0.60 \mathrm{~m}: \quad 0$ điểm $\quad 0 \%$

Tổng số điểm kiểm tra: 10198 điểm

Kết quả đánh giá ở trên cho thấy, số liệu đo sâu đảm bảo công tác thành lập bản đồ địa hình tỷ lệ 1:5.000.

\section{b. Với mảnh bản đồ tỷ lệ 1:10.000}

Tổng hợp kết quả độ lệch độ sâu giữa các tuyến đo sâu và đo kiểm tra như sau:

Tổng số điểm giao cắt kiểm tra là 501 điểm với:

- Số điểm nội suy có độ sâu từ 0 đến $-30 \mathrm{~m}$ là 70 điểm trong đó:

Số điểm có dh từ 0 đến $0,51 \mathrm{~m}$ là 66 điểm chiếm $13,17 \%$.

Số điểm có $\mathrm{dh}$ từ $0,51 \mathrm{~m}$ đến $0,60 \mathrm{~m}$ là 01 điểm chiếm $0,2 \%$.

Số điểm có dh lớn hơn $0,60 \mathrm{~m}$ là 03 điểm chiếm $0,6 \%$.

- Số điểm nội suy có độ sâu nhỏ hơn $-30 \mathrm{~m}$ có 431 điểm với đặc điểm:

Số điểm có $\mathrm{dh}$ từ 0 đến $0,51 \mathrm{~m}$ là 417 điểm chiếm $83,23 \%$.

Số điểm có $\mathrm{dh}$ từ $0,51 \mathrm{~m}$ đến $0,60 \mathrm{~m}$ là 01 điểm chiếm $0,2 \%$.

Số điểm có dh lớn hơn $0,60 \mathrm{~m}$ là 14 điểm chiếm $2,79 \%$.

Kết quả đánh giá trên cho thấy, số liệu đo sâu đảm bảo công tác thành lập bản đồ địa hình tỷ lệ 1:10.000.

\section{c. Với mảnh bản đồ tỷ lệ 1:50.000}

Tổng hợp kết quả độ lệch độ sâu giữa các tuyến đo sâu và đo kiểm tra như sau: 
Tổng số điểm giao cắt kiểm tra là 400 điểm với $100 \%$ số điểm giao cắt có độ sâu nằm trong khoảng từ 0 đến $-30 \mathrm{~m}$ với đặc điểm:

Số điểm có $\mathrm{dh}$ từ 0 đến $0,51 \mathrm{~m}$ là 389 điểm chiếm $97,25 \%$.

Số điểm có dh từ $0,51 \mathrm{~m}$ đến $0,60 \mathrm{~m}$ là 03 điểm chiếm $0,75 \%$.

Số điểm có dh lớn hơn $0,60 \mathrm{~m}$ là 08 điểm chiếm $2,0 \%$.

Kết quả đánh giá trên cho thấy, số liệu đo sâu đảm bảo công tác thành lập bản đồ địa hình tỷ lệ 1:50.000.

Kết quả tính thực nghiệm nêu trên đã được so sánh với số liệu đã được nghiệm thu và các Báo cáo tổng kết, nghiệm thu công trình với các sản phẩm đảm bảo yêu cầu như trong bản thuyết minh. Điều đó chứng tỏ tính đúng đắn của lý thuyết lựa chọn cũng như chương trình đã xây dựng được.

\section{Kết luận và kiến nghị}

Kết quả tính thực nghiệm như đã trình bày ở trên cho thấy, thuật toán và quy trình kiểm tra số liệu đo sâu bằng máy đo sâu hồi âm đơn tia đã lựa chọn hoàn toàn hợp lý. Chương trình kiểm tra dữ liệu đo sâu đã xây dựng hoàn toàn đáp ứng yêu cầu kiểm tra số liệu đo sâu bằng máy đo sâu hồi âm đơn tia phục vụ thành lập bản đồ địa hình đáy biển.

Kiểm tra số liệu đo sâu bằng công nghệ đo sâu hồi âm đa tia, do mật độ điểm đo sâu lớn, nên phương pháp đánh giá độ chính xác phái tiếp cận theo phương pháp khác nhau. Vấn đề này sẽ được trình bày trong bài báo tiếp theo. $\mathrm{O}$

\section{Tài liệu tham khảo}

[1]. Quy định cơ sở toán học, độ chính xác, nội dung và ký hiệu bản đồ địa hình đáy biển tỷ lệ 1:10000, (1998).

[2]. Quyết định số 03/2007/QĐ-BTNMT ngày 12 tháng 02 năm 2007 Quy định kỹ thuật thành lập bản đồ địa hình đáy biển tỷ lệ 1:50.000.

[3]. Thông tư số 24/2010/TT-BTNMT ngày 27/10/2010 về Quy định về đo đạc, thành lập bản đồ địa hình đáy biển bằng máy đo sâu hồi âm đa tia.

[4]. Nguyễn Văn Cương (2019), Báo cáo tổng hợp đề tài khoa học và công nghệ cấp cơ sở "Nghiên cứu, đề xuất quy trình đánh giá độ chính xác kêt quả đo sâu và xây dựng mô đun thực hiện quy trình đánh giá", Tông cục Biển hải đảo Việt Nam.

[5]. Nguyễn Văn Cương (2020), Nghiên cứu quy luật biến đổi vận tốc âm và hoàn thiện quy trình lấy mầu xác định vận tốc ậm trong môi trường nước biển khu vực Vịnh Bắc Bộ Việt Nam phục vụ khai thác hiệu quả các thiết bị thủy âm, Luận án tiến sỹ Kỹ thuật trắc địa - bản đồ, Trường Đại học Mỏ - Địa chất.

[6]. Vũ Hồng Tập, Nghiên cứu ảnh hưởng của việc xác định vận tốc âm đến kết quả đo sâu trong quy trình đo vẽ thành lập bản đồ địa hình đáy biển ở Việt Nam. Luận văn Thạc sỹ Kỹ thuật, Đại học Mỏ - Địa chất Hà Nội, (2011).

[7]. Dương Quốc Lương, Ứng dụng máy đo vận tốc âm trong công tác đo vẽ bản đồ địa hình đáy biển. Đề tài khoa học công nghệ, (2006).

[8]. Phạm Vũ Vinh Quang, Nghiên cứu ứng dụng công nghệ dò thủy âm quét sườn trong công tác thành lập bản đồ địa hình đáy biển ở Việt Nam. Luận văn Thạc sỹ Kỹ thuật, Đại học Mỏ - Địa chất Hà Nội, (2013).

[9]. Tăng Quốc Cương, Nghiên cứu phương pháp xác định và hiệu chỉnh độ nghiêng của đầu biến âm máy đo sâu hồi âm đơn tia trong đo đạc độ sâu đáy biển, Đề tài khoa học công nghê, (2013). O

(Xem tiếp trang 50) 